\title{
Prognostic Value of p53 Expression in Intestinal Neoplasms - a Study in a Tertiary Health Care Centre
}

\author{
Arputham Arunachalam ${ }^{1}$, Babiya Infant Arockiasamy ${ }^{2}$ \\ ${ }^{I}$ Department of Pathology, Thanjavur Medical College, Thanjavur \\ ${ }^{2}$ Department of Pathology, Thanjavur Medical College, Thanjavur
}

\begin{abstract}
Colorectal cancer is one of the most common tumours of the world with the varying distribution. Though small intestine is an uncommon site for tumour, it accounts for significant number of gastro-intestinal malignancies. Pathological assessment of the resected specimen is mandatory for assessing the postoperative outcome and the rationale for adjuvant therapy. Among the various prognostic factors, Mutation of the p53 tumour suppressor gene, plays an important role in the progression of colorectal carcinoma and so it represents a clinically useful marker of prognosis. The present study evaluates the role of p53 in intestinal neoplasms by assessing its level of expression and to correlate the level of p53 expression with the stage of the colorectal adenocarcinomas so that its prognostic value in colorectal adenocarcinomas can be calculated.
\end{abstract}

Keywords :Intestinal malignancies, colorectal adenocarcinoma, guardian genome, p53 expression, prognostic marker

\section{Introduction}

Colorectal cancer ranks second among the most common tumours of the world according to world cancer report $2000^{[1]}$. There is worldwide variation in the distribution of intestinal neoplasm, which appear largely due to exogenous factors rather than genetic. Cancer of the colon is not a very common disease in our country and the incidence is much lower than in the western world. The incidence in India is about $7 / 100000^{[2]}$.

Curiously the small intestine is an uncommon site for tumour, despite its great length and vast pool of dividing cells. Small bowel tumours account for $1-2 \%$ of all gastrointestinal neoplasms. ${ }^{[3]}$

Surgical resection is the primary treatment modality for colorectal carcinoma and the pathological assessment of the resection specimen provides data that is essential for patient management including the estimation of postoperative outcome and the rationale for adjuvant therapy. The essential elements of the pathological assessment of colorectal carcinoma resection specimen include pathological determination of TNM stage, tumour type, histological grade, status of resection margin and vascular invasion ${ }^{[4]}$.

The Tp53 gene located in chromosome 17p13.1 is the single most common target for genetic alteration in human tumours. As it plays an important role in cell cycle control and in the induction of apoptosis, it has been described as "the guardian of the genome", "the guardian angel gene", and "the master watchman" referring to its role in conserving stability by preventing genome mutation. This p53 tumour suppressor gene is mutated in approximately $70-80 \%$ of colorectal carcinoma. Mutation or loss of p53 usually occurs at the time of transition from adenoma to carcinoma in the adenoma-carcinoma sequence ${ }^{[5]}$. The frequency of p53 abnormalities increases with the progression of the lesion. Mutation of the p53 tumour suppressor gene is thought to play an important role in the progression of colorectal carcinoma and might therefore represent a clinically useful marker of prognosis.

The present study evaluates the role of tumour suppressor gene protein p53 in intestinal neoplasms by assessing its level of expression of p53 in intestinal neoplasms and to correlate the level of p53 expression with the stage of the colorectal adenocarcinomas so that its prognostic value in colorectal adenocarcinomas can be calculated.

\section{Materials And Methods}

Out of 11891 cases reported totally at Thanjavur Medical College, 111 cases of intestinal neoplasms diagnosed during the period of January 2010 to June 2012 were included in this retrospective study. All the cases of intestinal malignancies irrespective of the age, sex, type, stage and grade were included and the specimens were fixed in $10 \%$ buffered neutral formalin and were subjected to routine histopathological

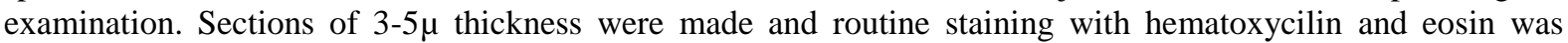
done.

A total of 30 cases of colorectal adenocarcinomas and 7 cases of small intestinal neoplasms were selected for studying the p53 expression. Formalin fixed paraffin embedded $4 \mu$ thick tissue sections were stained with antibody directed against p53. Staining was performed according to the standard immunohistochemical staining protocol. The percentages of p53 positive tumour cells were calculated by 
counting the number of brown stained tumour nuclei among the total number of cells in the most highly stained area in the selected microscopic fields at 400X magnification.

According to George E. Theodoropoulos et al p53 positivity was calculated ${ }^{[6]}$. If $\geq 10 \%$ or more of malignant nuclei were stained, the slide was scored as positive. While if fewer than $10 \%$ of the nuclei were stained, the slide was scored as negative. For small intestinal malignancy, all p53 positive nuclei were counted and the following semi-quantitative method was used: $[ \pm]-$ negative or equivocal $(<5 \%$ positive cells $),[+]-$ weaklmoderate $\left(5-50 \%\right.$ positivity) and $[++]$ - intensely positive $\left(>50 \%\right.$ positivity) ${ }^{[7]}$ The statistical analysis was performed to determine whether p53 protein expression was correlated with the stage of the colorectal adenocarcinomas. Statistical analysis was carried out with the aid of Chi-square test (Java script package). P value of less than 0.05 was taken to indicate statistical significance.

\section{Observation And Results}

\subsection{Incidence:}

TABLE - 1: Incidence of Intestinal Neoplasms in this Study

\begin{tabular}{|c|c|c|c|c|}
\hline Period & Total No. of specimens & Total neoplasms & Intestinal neoplasms & $\begin{array}{c}\text { Incidence } \\
(\%)\end{array}$ \\
\hline $\begin{array}{c}\text { Jan } 2010-\text { June } \\
2012\end{array}$ & 11891 & 4286 & 111 & 2.6 \\
\hline
\end{tabular}

Out of the 4286 neoplasms diagnosed totally, during the period of study (January 2010 - June 2012), there were 111 neoplasms arising in the intestine. Thus the annual incidence of intestinal neoplasms in our study was $2.6 \%$ (TABLE 1). Of 111 neoplasms, large intestinal neoplasm, with 100 cases, contributes significantly higher incidence with $90 \%$ (TABLE 2).

TABLE - 2: Incidence of Small and Large Intestine Neoplasms in this Study

\begin{tabular}{|c|c|c|c|c|}
\hline \multirow{2}{*}{ Total No. of neoplasms } & \multicolumn{2}{|c|}{ Small intestine } & \multicolumn{2}{c|}{ Large intestine } \\
\cline { 2 - 5 } & No. of cases & \% & No. of cases & \% \\
\hline 111 & 11 & 10 & 100 & 90 \\
\hline
\end{tabular}

3.2 Analysis of p53 Expression:

TABLE - 3: p53 Expression in Colorectal Adenocarcinoma in relation to histological type

\begin{tabular}{|c|c|c|c|c|c|}
\hline \multirow{2}{*}{ ADENOCARCINOMA } & \multicolumn{3}{|c|}{ p53 } & \multicolumn{2}{|c|}{ TOTAL } \\
\cline { 2 - 6 } & + & $\boldsymbol{\%}$ & - & \multicolumn{2}{c|}{} \\
\hline CONVENTIONAL ADENOCARCINOMA & 17 & 65 & 9 & 35 \\
\hline MUCINOUS ADENOCARCINOMA & 1 & 25 & 3 & 75 & 4 \\
\hline TOTAL & 18 & 60 & 12 & 40 & 30 \\
\hline
\end{tabular}

In this study $53.3 \%$ of the colorectal adenocarcinomas showed p53 positivity. p53 over expression was more frequent in non-mucinous adenocarcinomas than in mucinous adenocarcinomas (TABLE- 3 ).

TABLE - 4: p53 Expression in Colorectal Adenocarcinomas in Relation to Stage

\begin{tabular}{|c|c|c|c|c|}
\hline \multirow{2}{*}{ STAGE } & \multicolumn{2}{|c|}{$\mathbf{p 5 3}$} & \multirow{2}{*}{ TOTAL } & \multirow{2}{*}{ POSITIVE CASES } \\
\cline { 2 - 4 } & + & - & 7 & 42.86 \\
\hline I & 3 & 4 & 7 & 42.86 \\
\hline III & 3 & 4 & 12 & 75 \\
\hline IV & 9 & 3 & 4 & 75 \\
\hline TOTAL & 3 & 12 & 30 & \multicolumn{2}{|c}{} \\
\hline
\end{tabular}

For the purpose of statistical analysis stages I and II were considered as low stage tumours and stages III and IV were considered as high stage. The proportion of positively reacting colorectal tumours increases as the stage progresses. Statistical analysis using Chi- square test was done which revealed a $\mathrm{P}$ value of $<0.05\left(\mathrm{X}^{2}\right.$ $=4.74)$ which is statistically significant. Hence p53 expression is significantly correlated with the stage of tumour in colorectal adenocarcinoma (TABLE- 4) (CHART- 1). 


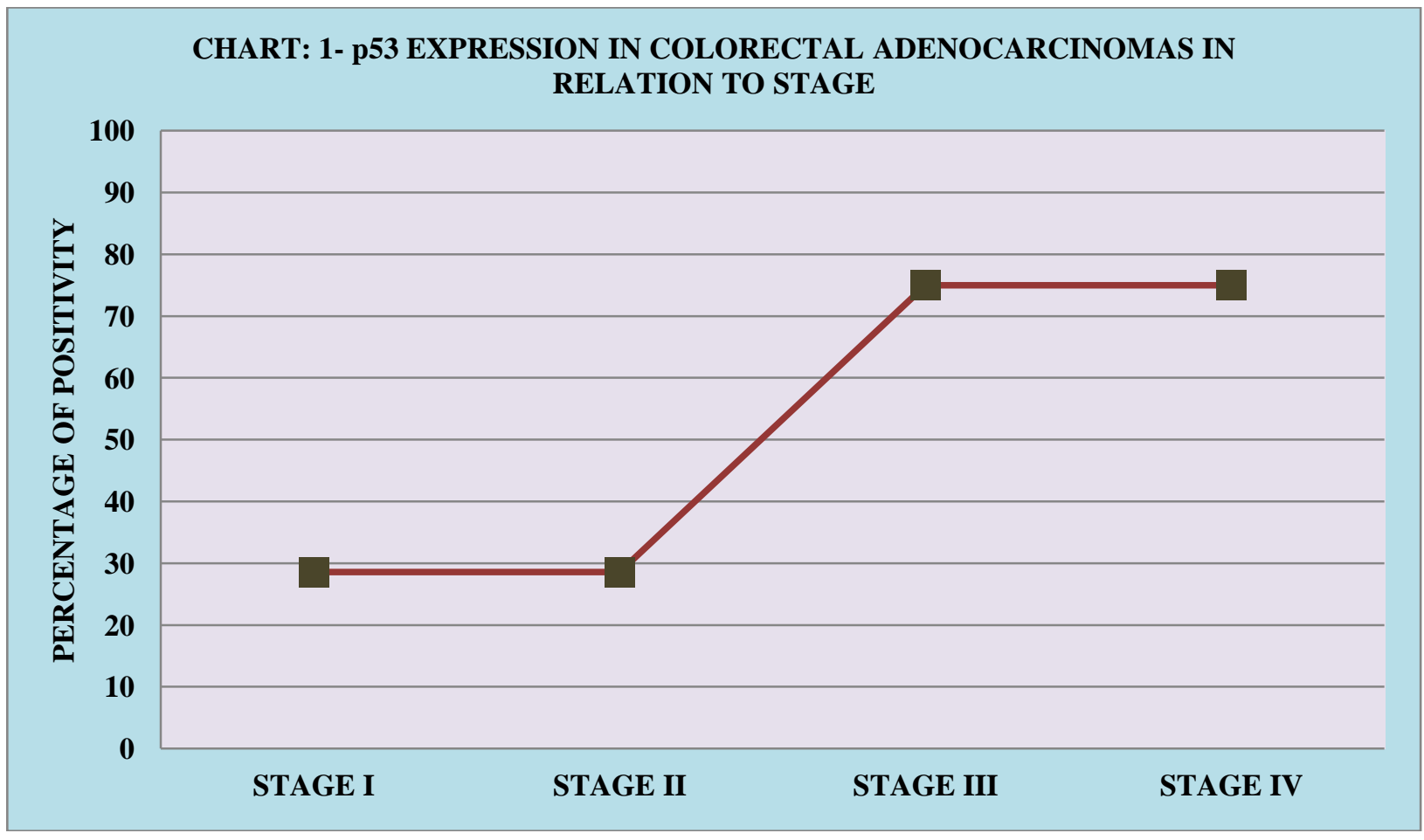

\begin{tabular}{|c|c|c|c|c|c|}
\hline \multirow{2}{*}{ TUMOURS } & \multicolumn{4}{|c|}{ p53 } & \multirow{2}{*}{ TOTAL } \\
\cline { 2 - 5 } & + & $\mathbf{\%}$ & - & $\%$ & 3 \\
\hline ADENOCARCINOMA & 2 & 67 & 1 & 33 & 2 \\
\hline LYMPHOMA & 1 & 50 & 1 & 50 & 2 \\
\hline
\end{tabular}

In this study $60 \%$ of the small intestinal tumours revealed p53 positivity. $67 \%$ of the adenocarcinomas and $50 \%$ of the lymphomas showed immune reactivity for p53 protein (TABLE-5).

DIAGRAMS: Well differentiated colorectal adenocarcinoma expressing p53 (score 0-4)

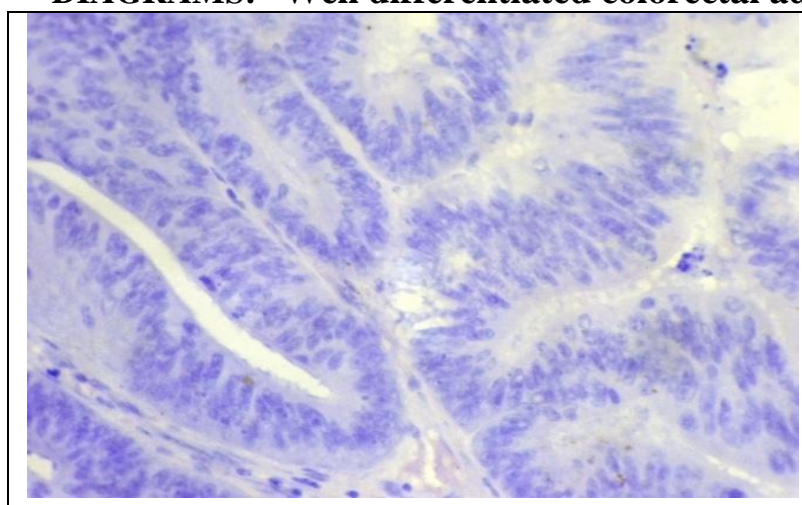

Figure 1: p 53- score 0

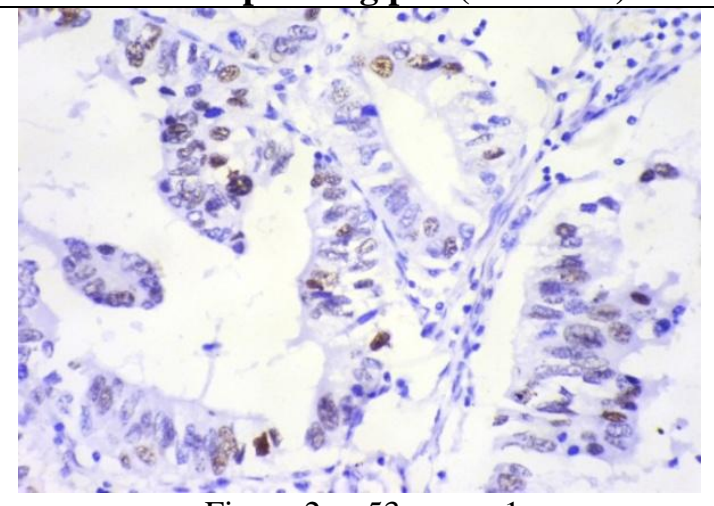

Figure 2: p 53- score 1 


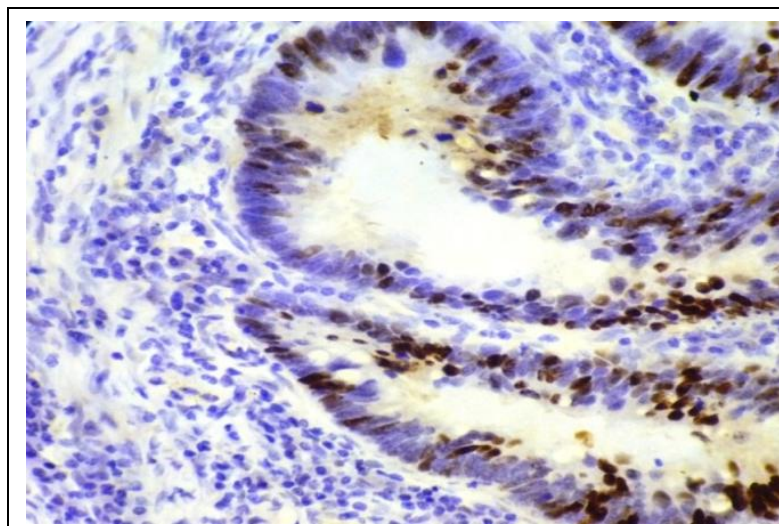

Figure 3: p 53- score 2

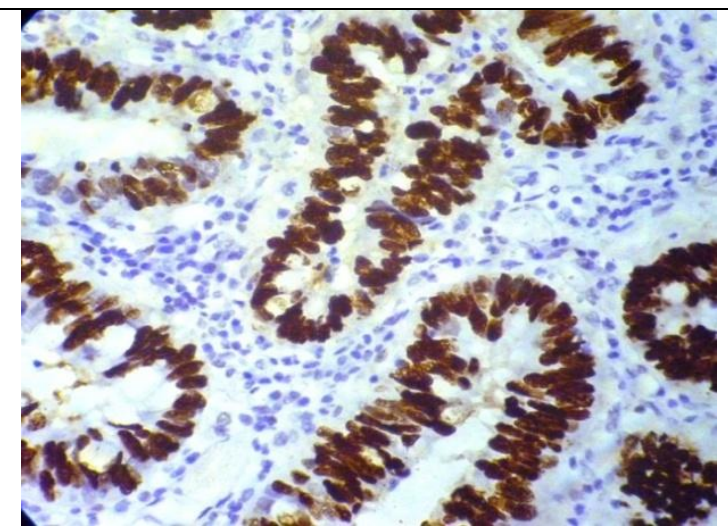

Figure 4: p 53- score 3

Moderately differentiated colorectal adenocarcinoma expressing p53 (score 0-4)

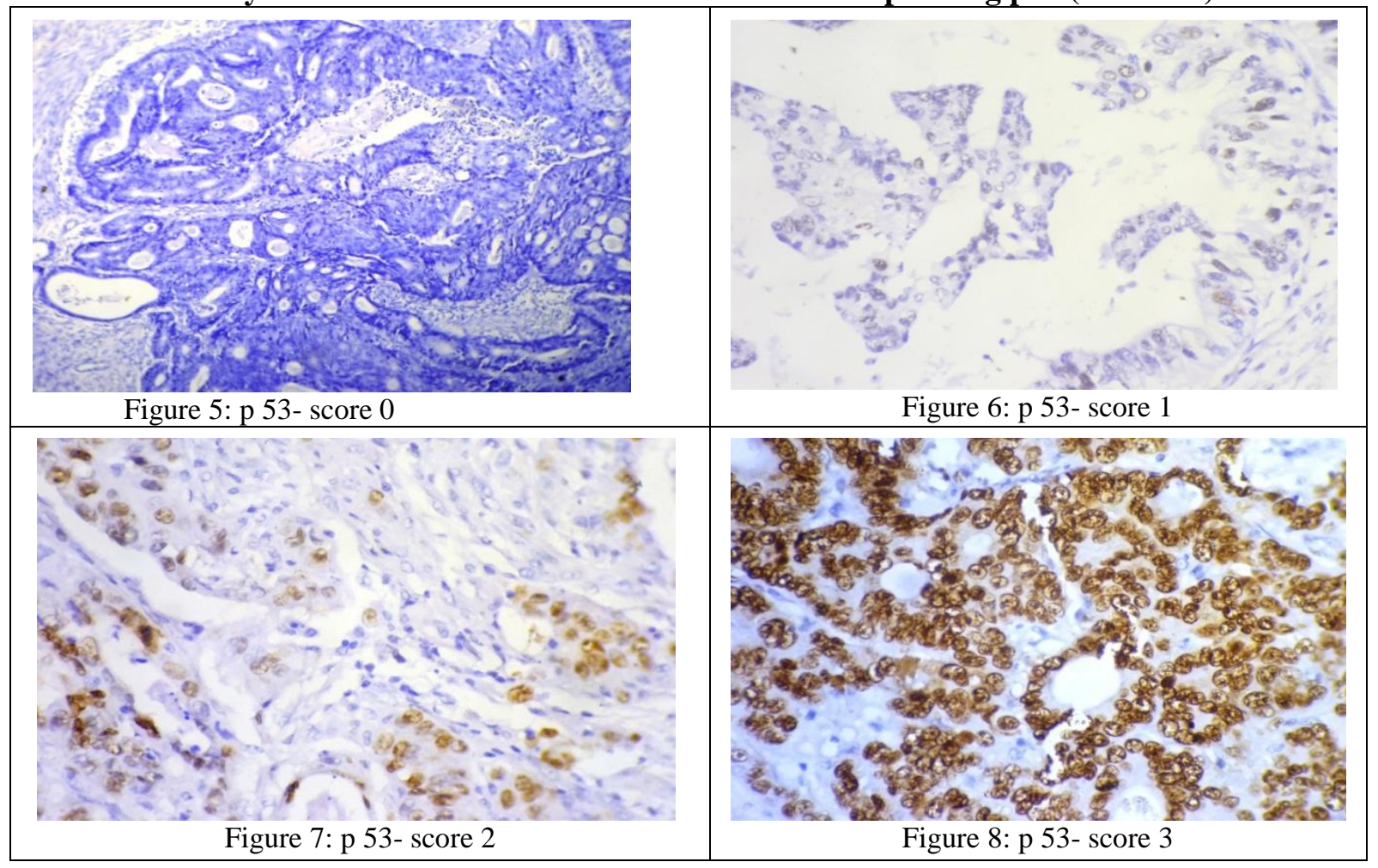

Poorly differentiated colorectal adenocarcinoma expressing p 53(score 0-4)

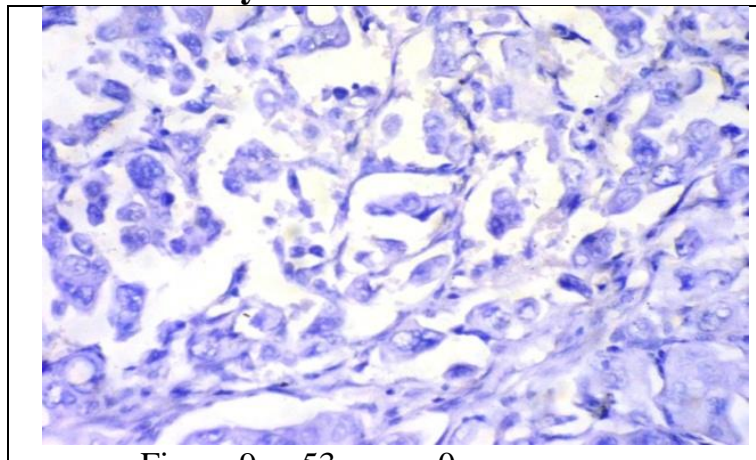

Figure 9: p 53- score 0

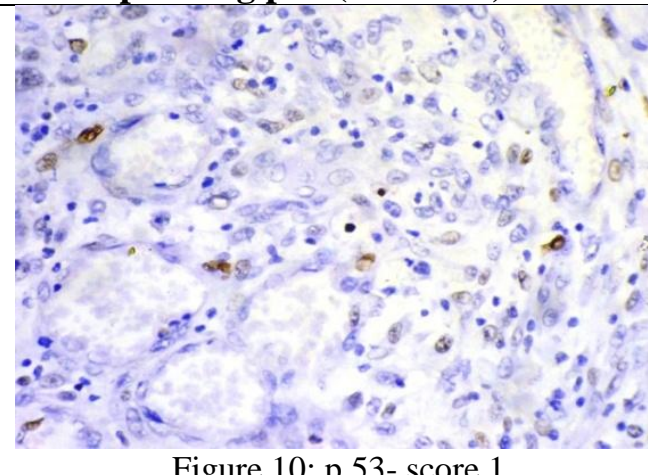

Figure 10: p 53- score 1 

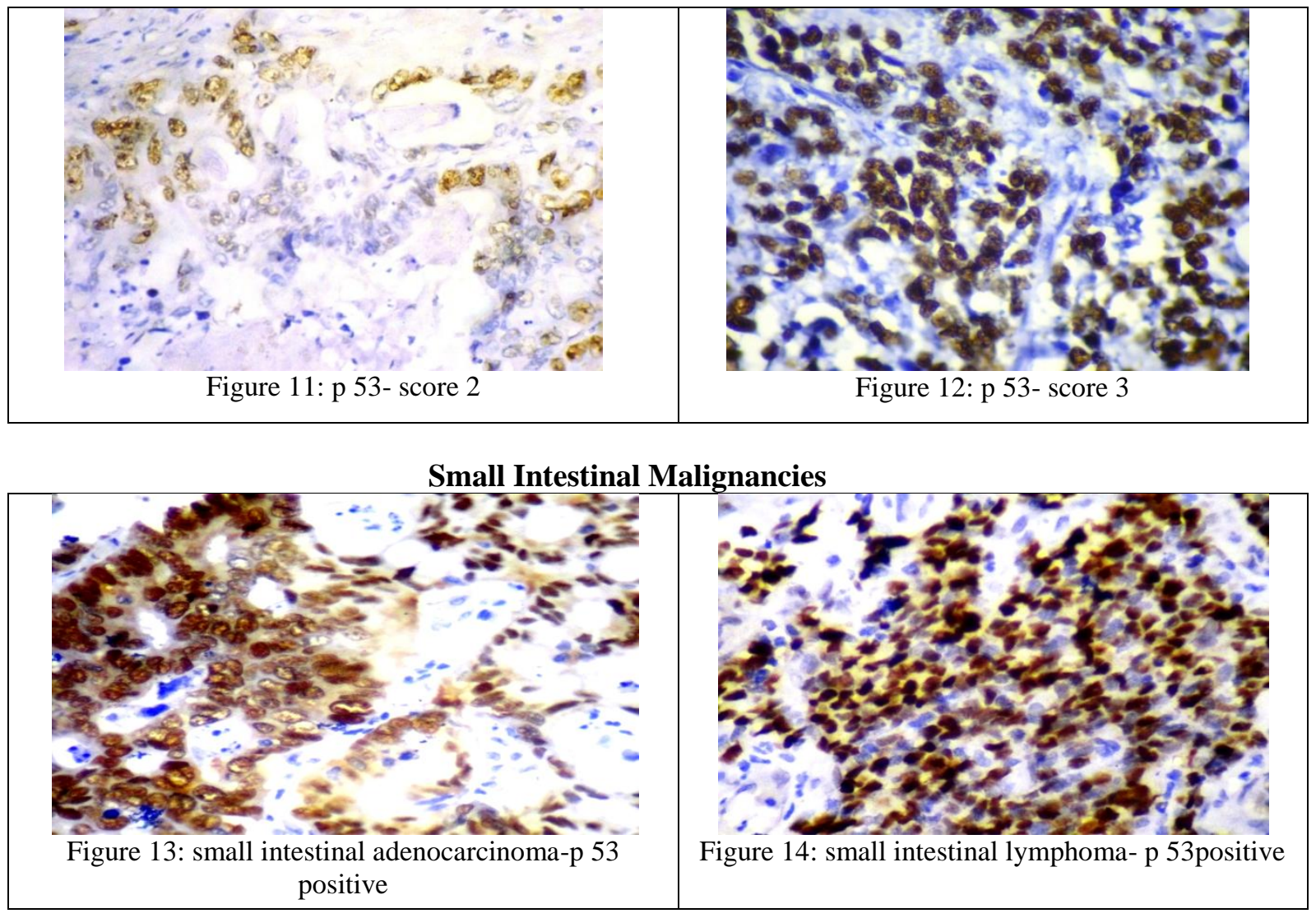

\section{1 p53 Immunoexpression in colorectal tumours:}

\section{Discussion}

p53 tumour suppressor gene is one of the most intensively studied tumour markers in the colorectal tumours. In this study 30 cases of colorectal carcinoma (colectomy specimens) were selected for the study of p53 expression by immunohistochemistry in various stages which included 4 mucinous adenocarcinomas. For the purpose of statistical analysis stages I and II were categorized as low stage and stages III and IV were categorized as high stage. According to George E Theodoropoulous et $\mathrm{a}^{[6]} \mathrm{p} 53$ immunostaining was done and the results were tabulated and analysed.

In the present study p53 expression was more frequent in non-mucinous adenocarcinomas $(57.7 \% \mathrm{Vs}$ $25 \%$ ). (TABLE-3) This is in accordance with the study by Yuan-Tzu-Lan et $\mathrm{a}^{[8]}$ who found that $39 \%$ of nonmucinous adenocarcinomas and $29 \%$ of mucinous adenocarcinomas showed p53 positivity and suggesting two different pathways, chromosomal instability and microsatellite instability of colorectal carcinogenesis respectively. T Starzynska et $\mathrm{al}^{[9]}$ studied the relation of $\mathrm{p} 53$ expression in colorectal carcinoma with histological, clinical, prognostic features using follow-up data and concluded that p53 expression occurred as a late event and was associated significantly associated with advanced stage of disease, early relapse and death. J Walker et $\mathrm{al}^{[10]}$ in their study found that the stage is the most accurate prognostic factor for survival. They concluded that p53 overexpression in colorectal carcinoma correlated with poor prognosis. In this study the proportion of $\mathrm{p} 53$ nuclear protein expression increases as the stage progresses. This association was proved to be statistically significant by using chi square test $(\mathrm{P}<0.05)$. This is in accordance with the study done by $\mathrm{T}$ Starzynska et al ${ }^{[9]}$ George E Theodoropoulous et al. ${ }^{[6]}$ Several previously published series have supported the deleterious effect of $\mathrm{p} 53$ over expression. ${ }^{[11,12]}$

Scott $\mathrm{N}$ et al ${ }^{[13]}$ analysed 52 colorectal carcinomas in their study and found no correlation with p53 overexpression and several factors related to prognosis.

Campo et al (1991) ${ }^{[14]}$ also, did not find relationship between p53 expression and degree of differentiation and stage of the tumour, concluded that p53 is not a good prognostic indicator in large bowel malignancy. The discrepancies in the prognostic role of p53 in colorectal adenocarcinomas might be a result of different kind of materials, methods and antibodies used or differences in the number of tumours examined.

p53 expression increases as the stage progresses, stage is the established prognostic factor in colorectal carcinoma, and also there is significant correlation between high p53 levels and stage of the colorectal carcinomas, so it can be used as a prognostic marker to assess the invasiveness and metastatic potential of the colorectal tu 


\begin{tabular}{|c|c|c|}
\hline S.NO & STUDIES & $\begin{array}{l}\text { p53 EXPRESSION } \\
(\%)\end{array}$ \\
\hline 1. & Mohammad - Reza et al $^{[15]}$ & 59 \\
\hline 2. & 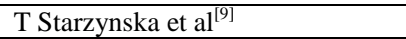 & 46 \\
\hline 3. & Scott $\mathrm{N}$ et $\mathrm{al}^{[13]}$ & 42 \\
\hline 4. & O Petrisor et al $^{[16]}$ & 66 \\
\hline 5. & George E Theodoropoulous et $\mathrm{al}^{[6]}$ & 63.4 \\
\hline 6. & Present study & 53.3 \\
\hline
\end{tabular}

In our study $53.3 \%$ of colorectal adenocarcinomas showed p53 nuclear protein overexpression. These differences may be due to the use of different scoring system and inter-observer variability. (TABLE- 6)

\section{2 p53 Immunoexpression - Small Intestine:}

p53 expression was studied in 5 small intestinal tumours of which 3 cases were adenocarcinoma and 2 cases were lymphomas. p53 expression was quantified according to DA Spandidios et al ${ }^{[7]}$ and results were tabulated and analysed. In the present study p53 expression is seen in $60 \%$ of small intestinal tumours. Of 3 cases of adenocarcinoma 2 cases (67\%) were p53 positive and 1 out of 2 cases $(50 \%)$ of lymphoma were positive for p53. Intense positivity were seen in lymphomas ( $>50 \%$ of cells). This is in accordance with the study done by DA Spandidos et al. ${ }^{[7]}$

DA Spandidos et al ${ }^{[7]}$ found immunoreactivity in $46 \%$ of small intestinal tumours. Ge C, He S, Tian $\mathrm{Y}^{[17]}$ reported nuclear positivity for $\mathrm{p} 53$ in $75 \%$ of small intestinal tumours. Tadashi Terada ${ }^{[18]}$ found $51.21 \%$ of p53 positivity in small intestinal tumours. M Svrcek et al ${ }^{[19]}$ found $\mathrm{p} 53$ positivity in $51.85 \%$ of small intestinal adenocarcinoma. Krugmann et $\mathrm{al}^{[20]}$ reported p53 overexpression in $75 \%$ of small intestinal lymphomas. These differences may be due to the use of different scoring system and inter-observer variability.

D A Spandidos et al ${ }^{[7]}$ concluded that $\mathrm{p} 53$ gene may be involved in the pathogenesis of small intestinal tumours. Tadashi Terada, ${ }^{[18]}$ in his study, found that $\mathrm{p} 53$ protein overexpression was present in most of the primary small intestinal carcinomas. Ge C et al ${ }^{[17]}$ in their study showed that the degree of p53 protein expression was significantly correlating with the grade of the tumour cell differentiation, invasion, metastasis and prognosis. According to Krugmann et $\mathrm{al}^{[20]}$ and Ming Qing et $\mathrm{al}^{[21]} \mathrm{p} 53$ overexpression in small intestinal lymphomas is a critical factor in the transformation of low grade MALT lymphoma and the overexpression also indicate the aggressive behavior of high grade tumours.

p53 immunoexpression was seen in both small intestinal adenocarcinoma and lymphoma. Intense positivity was observed in small intestinal lymphoma. This shows the involvement of p53 gene in the pathogenesis of small intestinal tumours. Due to low sample size, the prognostic value of p53 could not be determined.

\section{Conclusion}

In colorectal tumours p53 expression increases as the stage progresses and also there is significant correlation between high p53 levels and stage of colorectal carcinomas. So it can be used as a prognostic marker to assess the invasiveness and metastatic potential of the colorectal tumours.

There is a statistically significant correlation between $\mathrm{p} 53$ protein expression and stage of the colorectal cancer and hence p53 immunoreactivity is an important independent prognostic marker in patients with colorectal carcinoma. P53 protein overexpression has been associated with a worst overall survival after cancer diagnosis. Considering the acceptable reliability and feasibility of detection of p53 by immunohistochemistry, this marker may be expected to serve as a new genetic marker for predicting recurrence and response to chemotherapy in patients with colorectal cancer.

\section{Reference}

[1] Norco masukura, Hicoko ohgaki, Rens Lambert. World cancer report. Lyon, IARC press, 2003;194-202.

[2] Desai PB. Carcinoma of the colon. Bombay hospital journal 2000;42:256-9.

[3] Hatzaras I, Palesty JA, Abir F et al. Small bowel tumours. Epidemiologic and clinical characteristics of 1,260 cases from the Connecticut tumour registry. Arch surg 2007;142:229.

[4] Crompton CC. Colorectal carcinoma Diagnostic, prognostic and molecular features. Mod Pathol 2003;16:376-88

[5] Chen Liu, Jame M. Grawford. The Gastrointestinal tract. In Robins and Cotran. Pathologic basis of disease, $8^{\text {th }}$ edition. Philadelphia, Elsevier, 2010;822-25.

[6] George E. Theodoropoulos, Eleni Karafoka, Joanna G. Papailiou. p53 and EFGR expression in colorectal cancer: A reappraisal of 'old' tissue markers in patients with long follow-up. Anti-cancer research 2009;29:785-92.

[7] DA Spandidos, V Zoumpourlis, V Gorgoulis. p53 expression in human small intestinal tumours. Oncology reports 1994;1:885-7.

[8] Yuan-Tzu Lan, Shin-Ching-Chang, Anna Fen-Yau Li. p53 protein accumulation as a prognostic marker in sporadic colorectal cancer. Int J Colorectal Dis 2007;22:499-506.

[9] T. Starzynska, M. Bromley, A. Ghosh. Prognostic significance of p53 overexpression in gastric and colorectal carcinoma. Br J Cancer 1992;66:558-62. 
[10] J Walker, P Quirke. Prognosis and response to therapy in colorectal carcinoma. Eur J Cancer 2002;38:880-86.

[11] Gallego MG, Acenero MJ, Ortega S et al. Prognostic influence of p53 nuclear overexpression in colorectal carcinoma. Dis Colon Rectum 2000;43:971-5.

[12] Gon HS, Elnatan J, Low CH, Smith DR. p53 point mutation and survival in colorectal carcinoma patients: Effect of disease dissemination and tumour location. Int J Oncol 1999;15:491-8

[13] Scott N, Sagar P, Stewart et al. p53 in colorectal carcinoma: Clinicopathological correlation and prognostic significance. Br J cancer 1991;63:317.

[14] Campo E, De la Calle-Martin, O Miquel et al. Loss of heterozygosity of p53 gene and protein expression in human colorectal carcinomas. Cancer Res 51:4436.

[15] Mohammad-Reza, Ghavam-Nasiri, Kamran Ghafarzadegan, Mehdi Seilanian-Toosi. Expression of p53 in colorectal carcinoma: Correlation with clinicopathologic features. Arch Iranian Med 2007;10:38-42.

[16] O Petrisor, Simona Eliza Giusca, Maria Sajin et al. Ki-67, p53 and bcl-2 analysis in colonic versus rectal adenocarcinoma. Romanian Journal of Morphology and Embryology 2008;49:163-71.

[17] Ge C, He S, Tian Y. Study of p53 protein expression in cancers of the small intestine and its relationship to clinical course and prognosis. 1996;18:279-81.

[18] Tadashi Terada. Malignant tumours of the small intestine: A histopathologic study of 41 cases among 1,312 consecutive specimens of small intestine. Int J Clin Exp Pathol 2012;5:203-9.

[19] M Svrcek, F Jourdan, N Sebbagh et al. Immunohistochemical analysis of adenocarcinoma of the small intestine: A tissue microarray study. J Clin Pathol 2003;56:898-903.

[20] Krugmann J, Dirnhofer S, Gschwendther A et al. Primary gastrointestinal B-cell lymphoma - A clinicopathological immunohistochemical study of 61 cases with an evaluation of prognostic parameters. Pathol Res Pract 2001;197:385-93.

[21] Ming Qing DU, Huaizheng Peng, Nalini Singh et al. The accumulation of p53 abnormalities is associated with progression of mucosa associated lymphoid tissue lymphoma. Blood Journal 1995;86:4587-93. 